\title{
Adenoma pleomorfo vs lesión linfoepitelial benigna. Diagnóstico clínico e histopatológico
}

Pleomorphic adenoma vs benign lymphoepithelial cyst. Clinical and histopathological diagnosis

\author{
Adenoma pleomórfico vs lesão linfoepitelial benigna. \\ Diagnóstico clínico e histopatológico
}

\author{
Samuel E. Urbano Del Valle ${ }^{1}$ \\ Eilien G. Tovío Martínez ${ }^{2}$ \\ Shanny L. Miranda Duncan ${ }^{3}$ \\ Jonathan Harris Ricardo ${ }^{4}$
}

Recibido: 20 de diciembre de 2019

Aprobado: 31 de agosto de 2020 Publicado: 15 de noviembre de 2020

Cómo citar este artículo:

Urbano-DelValle SE, Tovío-Martínez EG, Miranda-Duncan SL, Ricardo JH. Adenoma pleomorfo vs lesión linfoepitelial benigna. Diagnóstico clínico e histopatológico. Revista Nacional de Odontología. (2020); 16(2), 1-13. doi: https://doi.org/10.16925/2357-4607.2020.02.12

Reporte de caso. https://doi.org/10.16925/2357-4607.2020.02.12

1 DDS, Estomatólogo y Cirujano Oral de la Universidad de Cartagena, Residente $2^{\circ}$ año de Cirugía Oral y Maxilofacial de la Universidad de Antioquía-Medellín, Colombia, Grupo GITOUC. Facultad de Odontología, Universidad de Cartagena. Barrio Zaragocilla Campus de la Salud. Cartagena. D.T. y C. Colombia. Suramérica.

Correo electrónico: surbanod@unicartagena.edu.co.

ORCID: https://orcid.org/0000-0002-5773-8642

2 DDS, Especialista en Estomatología y Cirugía Oral, Grupo GITOUC, Facultad de Odontología, Universidad de Cartagena, Colombia.

ORCID: https://orcid.org/0000-0003-3702-2791

3 DDS, Odontóloga. Universidad de Cartagena, Colombia. Semilleros de investigación. ORCID: https://orcid.org/0000-0003-1797-3346

4 Odontólogo. MSc en Microbiología Molecular. Especialista en Estomatología y Cirugía Oral. Hospital Naval de Cartagena. Docente Corporación Universitaria Rafael Núñez, Universidad de Cartagena. Colombia.

ORCID: https://orcid.org/0000-0002-4662-0729 


\section{Resumen}

Introducción: el adenoma pleomorfo es la neoplasia de mayor incidencia de glándulas salivales, representando alrededor del 3-10 \% de los tumores de la región de cabeza. La lesión linfoepitelial benigna por otra parte ha sido ampliamente reconocida como una causa común de agrandamiento de la glándula parótida en pacientes por lo general infectados con VIH. Las lesiones quísticas de la glándula parótida son poco frecuentes, se caracterizan por ser tumores de crecimiento lento, asintomático, de consistencia firme y cubiertos por piel o mucosa sana.

Objetivo: describir las características histológicas, clínicas e imagenológicas, las cuales han de llevarse a cabo de manera multidisciplinaria, para realizar un plan de tratamiento adecuado y acertado de la patología.

Caso clínico: paciente masculino que asiste a consulta por presentar lesión tumoral asintomática, ubicada en región preauricular derecha. Se envía resonancia magnética de la zona confirmando la lesión, se realiza abordaje quirúrgico para eliminación de la lesión y tejidos comprometidos. Posteriormente, estudio histológico confirma el diagnóstico de adenoma pleomorfo.

Conclusión: Aunque la intervención quirúrgica es el tratamiento de elección tanto del adenoma pleomorfo como de la lesión linfoepitelial benigna, se hace imperativo realizar un exhaustivo estudio clínico, imagenológico e histopatológico de la posible lesión, y así descartar otras entidades que requieran un plan de tratamiento totalmente distinto, y así evitar cometer errores.

Palabras clave: adenoma, glándula parótida, glándulas salivales, quiste branquial, adenolinfoma, histología (DeCS).

\section{Abstract}

Introduction: the pleomorphic adenoma is the neoplasm with the highest incidence of salivary glands, representing around $3-10 \%$ of the tumors of the head region; the benign lymphoepithelial lesion, on the other hand, has been widely recognized as a common cause of enlarged parotid gland in patients usually infected with HIV. The cystic lesions of the parotid gland are rare, characterized by being slow-growing, asymptomatic tumors of firm consistency and covered by healthy skin or mucosa.

Objective: to describe the histological, clinical and imaging characteristics, which must be carried out in a multidisciplinary manner, in order to carry out an appropriate and accurate treatment plan for the pathology.

Clinical case: male patient attending the clinic due to asymptomatic tumor lesion located in the right preauricular region. Magnetic resonance of the area is sent confirming the lesion, a surgical approach is made to eliminate the injury and compromised tissues, subsequently, histological study confirms the diagnosis of pleomorphic adenoma.

Conclusion: Although surgical intervention is the treatment of choice for both, the pleomorphic adenoma and the benign lymphoepithelial lesion, it is imperative to perform an exhaustive clinical, imaging and histopathological study of the possible lesion, thus ruling out other entities that require a treatment plan totally different, avoiding mistake.

Keywords: Adenoma, Parotid Gland, Salivary Glands, Branchial Cyst, Adenolymphoma, Histology (MeSH).

\section{Resumo}

Introdução: o adenoma pleomórfico é a neoplasia com maior incidência de glândulas salivares, representando cerca de $3-10 \%$ dos tumores da região da cabeça. A lesão linfoepitelial benigna, por outro lado, foi amplamente reconhecida como uma causa comum de aumento da glândula parótida em pacientes geralmente infectados pelo HIV. As lesões císticas da glândula parótida são incomuns, caracterizadas por serem tumores de crescimento lento, assintomáticos, de consistência firme e recobertos por pele ou mucosa sã. 
Samuel E. Urbano Del Valle, Eilien G. Tovío Martínez, Shanny L. Miranda Duncan, 3 Jonathan Harris Ricardo

Objetivo: descrever as características histológicas, clínicas e de imagem, que devem ser realizadas de forma multidisciplinar, a fim de realizar um plano de tratamento adequado e correto para a patologia.

Caso clínico: paciente masculino que comparece a consulta por lesão tumoral assintomática, localizada em região pré-auricular direita. Uma ressonância magnética da área é enviada confirmando a lesão, uma abordagem cirúrgica é realizada para remover a lesão e os tecidos comprometidos. Posteriormente, um estudo histológico confirma o diagnóstico de adenoma pleomórfico.

Conclusão: Embora a intervenção cirúrgica seja o tratamento de escolha tanto para o adenoma pleomórfico quanto para a lesão linfoepitelial benigna, é imprescindível a realização de um estudo clínico, de imagem e histopatológico exaustivo da possível lesão e, assim, descartar outras entidades que requeiram um plano de tratamento totalmente diferente, evitando assim cometer erros.

Palavras-chave: adenoma, glândula parótida, glândulas salivares, cisto branquial, adenolinfoma, histologia (DeCS).

\section{Introducción}

Los tumores de las glándulas salivales son poco frecuentes. Representan solo un 2 a $4 \%$ de las patologías desarrolladas en cabeza y cuello, siendo el adenoma pleomorfo el más común de los tumores benignos desarrollado en las glándulas salivales (1). Por otro lado, la lesión linfoepitelial benigna, es una lesión poco común, que se asocia principalmente a pacientes VIH positivos. Estas lesiones se desarrollan principalmente en la glándula parótida, aunque pueden afectar las otras glándulas salivales y el área cervical lateral (2).

El adenoma pleomorfo o también conocido como tumor mixto benigno, se presenta frecuentemente en la adultez, entre la segunda y cuarta década de la vida. Con mayor predominio en el sexo femenino, tiene una prevalencia entre el $60 \%$ y 89,5 \%. La localización más usual es en la glándula parótida en un 8 \% a diferencia de las glándulas salivales menores donde se encuentra con un porcentaje entre $18 \%$ y $50 \%$ (3).

La lesión linfoepitelial o también conocida como quiste branquial, es un tipo de lesión benigna de crecimiento lento que se presenta principalmente en adultos, con predilección del sexo femenino. Autores como Ahamed y col, han reportado que estas lesiones se encuentran, principalmente, en pacientes diagnosticados con VIH positivo, aunque actualmente la etiología de este tipo de lesión es controvertida (4).

Clínicamente el adenoma pleomorfo se desarrolla cómo un nódulo, de crecimiento lento, consistencia firme, bien delimitado, asintomático, recubierto por piel o mucosa clínicamente sana (5). El tumor no se adhiere a planos profundos, excepto cuando afecta el paladar duro, en estos casos puede extenderse hacia el periostio y el hueso subyacente. A la palpación puede tener cavidades quísticas, siendo en estos casos blandos (6). 
Por otro lado, las lesiones linfoepiteliales suelen aparecer como una inflamación difusa, de crecimiento lento, fluctuante, recubierto por piel o mucosa sana y asintomático. Este tipo de lesiones suelen asociarse especialmente con la glándula parótida. También aparece en áreas del cuello, se desarrolla debido a la dilatación ductular quística inducida por linfocitos y siempre se diagnóstica mediante examen histopatológico después de su extirpación (7).

Radiográficamente el adenoma pleomorfo se observa como una lesión radiopaca, circunscrita que compromete a la estructura donde esté ubicado. Para confirmar el diagnóstico es necesario complementarlos con otras ayudas diagnosticas como la PAAF (punción aspiración con aguja fina) con un 91,6 \% de precisión y otros como la biopsia incisional y tomografía computarizada (TC), para delimitar correctamente los límites (8). Solo el 6 \% representa potencial de transformación maligna del adenoma pleomorfo (9).

Por otro lado, la lesión linfoepitelial mediante un TC se observa cómo una lesión hipodensa, homogénea y sin realce. La resonancia magnética simple (IRM) puede mostrar una lesión quística bien definida, delgada y de pared lisa, lo que sugiere un alto contenido proteínico o hemorrágico sin componente sólido (10).

Histológicamente el adenoma pleomorfo es una neoplasia benigna con un componente epitelial, mioepitelial y estromal, de estos componentes es el estroma quien le da la distinción al adenoma pleomorfo. El patrón histopatológico del componente epitelial son células cuboidales, basales, escamosas, fusiformes, plasmocitoides. El componente de células mioepiteliales morfológicamente puede ser parecido a las células luminales o contener un citoplasma más claro con núcleos hipercromáticos (11). Por otra parte, el patrón histopatológico que se observa en la lesión linfoepitelial benigna es el de una hendidura revestida de epitelio glandular o escamosa rodeada por abundante tejido linfoide con centros germinales prominentes, con algunos especímenes que incluso contienen tejido y conductos de las glándulas salivales (12).

Cuando el adenoma pleomorfo es intraoral y afecta la mucosa bucal se debe hacer un diagnóstico diferencial principalmente con neurofribromas y otros tumores como la lesión linfoepitelial benigna. En lesiones de la glándula parótida se debe confirmar que no es una neoplasia, parotiditis crónica, quiste parotídeo o una hipertrofia parotídea asociada a diabetes mellitus (13).

El tratamiento de elección para el adenoma pleomorfo es la conservación de los nervios con una parotidectomía parcial o disección extracapsular. Hay que tener en cuenta que las recurrencias aparecen cuando la extirpación es incompleta (14). En el caso de la lesión linfoepitelial, el tratamiento a seguir es igualmente la extirpación 
completa con margen suficiente de tejido sano o si aparece en parótida por ejemplo, la parotidectomía superficial (10).

El objetivo del presente estudio consiste en describir las características histológicas, clínicas e imagenológicas, las cuales han de llevarse a cabo de manera multidisciplinaria para, de esta forma, realizar un tratamiento adecuado y acertado de la patología.

\section{Caso clínico}

Paciente masculino de 53 años de edad, que acude al servicio de Estomatología y Cirugía Oral de la Universidad de Cartagena. Presenta lesión tumoral localizada a nivel preauricular, acompañada de disminución en la apertura bucal. En el interrogatorio no se presentan antecedentes médico-familiares o personales relevantes para el caso.

Al examen físico general se observa lesión tumoral en región preauricular derecha, de 4 meses de evolución aproximadamente, de crecimiento lento, asintomático a la palpación, normocrómico con respecto al tejido adyacente, de consistencia resilente, de aproximadamente $3 \mathrm{~cm}$ de diámetro, no móvil, adherido a planos profundos y de etiología desconocida. Se observa asimetría hemifacial derecha, al examen intraoral se presenta hipometría oral de $30 \mathrm{~mm}$, con presencia de espacios edéntulos bimaxilares.

En resonancia magnética (RM), de región parotídea derecha, se observa compromiso del lóbulo superficial derecho de la parótida, hacia anterior se observa, una imagen ovoidea que mide 25×26×20 mm en sentido anteroposterior, cráneocaudal y transversal, lesión en general con márgenes definidos, delimitados por una cápsula hipointensa en influencia T1, iso e hiperintenso en T2. Los espacios masticatorios, parafaríngeos, prevertebral, carotídeos y parotídeo izquierdo sin alteraciones. El musculo masetero en la vecindad se define de bordes normales sin alteraciones de morfología o señal (figura 1 A y B). 


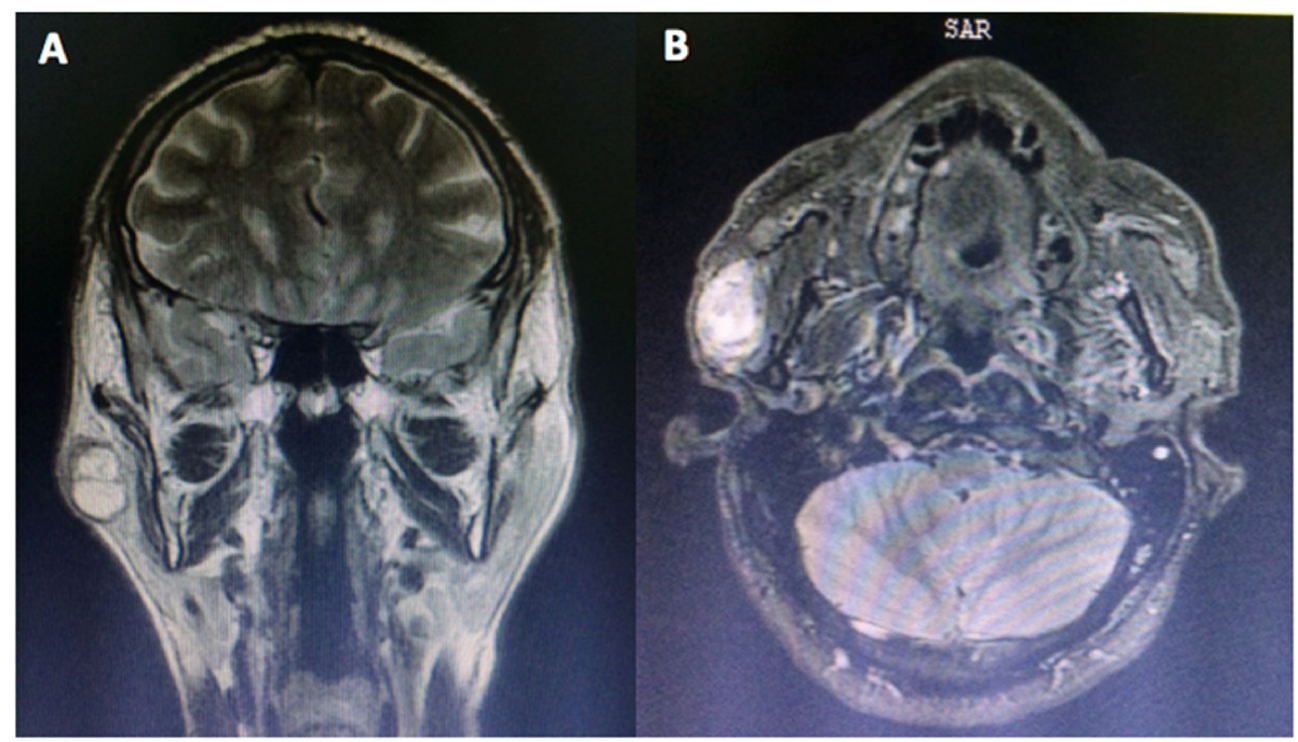

Figura 1A. Resonancia magnética de región parotídea derecha, se observa compromiso del lóbulo superficial derecho de la parótida, hacia anterior se observa, una imagen ovoidea que mide $25 \times 26 \times 20 \mathrm{~mm}$ en sentido anteroposterior, 1B. En corte transversal, lesión en general con márgenes definidos, delimitados por una cápsula hipointensa en influencia T1, iso e hiperintenso en T2.

Fuente: elaboración propia

Teniendo en cuenta la anamnesis, características clínicas e imagenológicas se realizó impresión clínica de adenoma pleomorfo, con diagnósticos diferenciales de lesión linfoepitelial benigna, lipoma o sialosis.

Una vez firmado el consentimiento informado, se lleva a cabo biopsia incisional, se envía muestra a estudio anatomopatológico. Sin embargo, los resultados no son concluyentes, por lo que se decide llevar a cabo una segunda toma de muestra, en la que refieren presencia de lesión linforreticular, con presencia de metaplasma ductal y atrofia acinar. Se decide llevar a cabo un nuevo estudio de placas en el Hospital Universitario del Caribe. Confirman el diagnóstico previo de adenoma pleomorfo, por todo ello, se decide realizar remoción quirúrgica de la lesión.

Previa asepsia y antisepsia, se lleva a cabo protocolo quirúrgico. A continuación, bajo anestesia general a través de intubación orotraqueal, se procede a realizar abordaje endaural de $4 \mathrm{~cm}$, se realiza disección por planos hasta hallar tumoración parotídea. Se evidencia unilobulada, encapsulada y vascularizada, se procede a realizar parotidectomía parcial, preservando el nervio facial, obteniendo lesión tumoral de aproximadamente $3.5 \times 3 \times 3$ centímetros de $100 \mathrm{gr}$. Se continua realizando hemostasia y se coloca dren tipo Penrose hacia región parotídea, para proceder con la sutura vycril 3.0 en plano profundo y nylon 5.0 en plano superficial, se concluye procedimiento sin complicaciones. Se envía muestra a estudio histopatológico con 
Samuel E. Urbano Del Valle, Eilien G. Tovío Martínez, Shanny L. Miranda Duncan, 7 Jonathan Harris Ricardo

resultado definitivo de neoplasia compuesta por células epiteliales que forman conductos entremezclados con células mioepiteliales, en el que se evidencia un estroma con áreas tanto mixoides como hialinizadas. A nivel periférico se halla capsula conformada por tejido conjuntivo denso, con presencia de infiltrado inflamatorio y bien vascularizado, además de células neoplásicas, subsanando los interrogantes iniciales y confirmando como diagnóstico, adenoma pleomorfo, pudiendo descartar la lesión linfoepitelial benigna (figura 2 y 3 ).

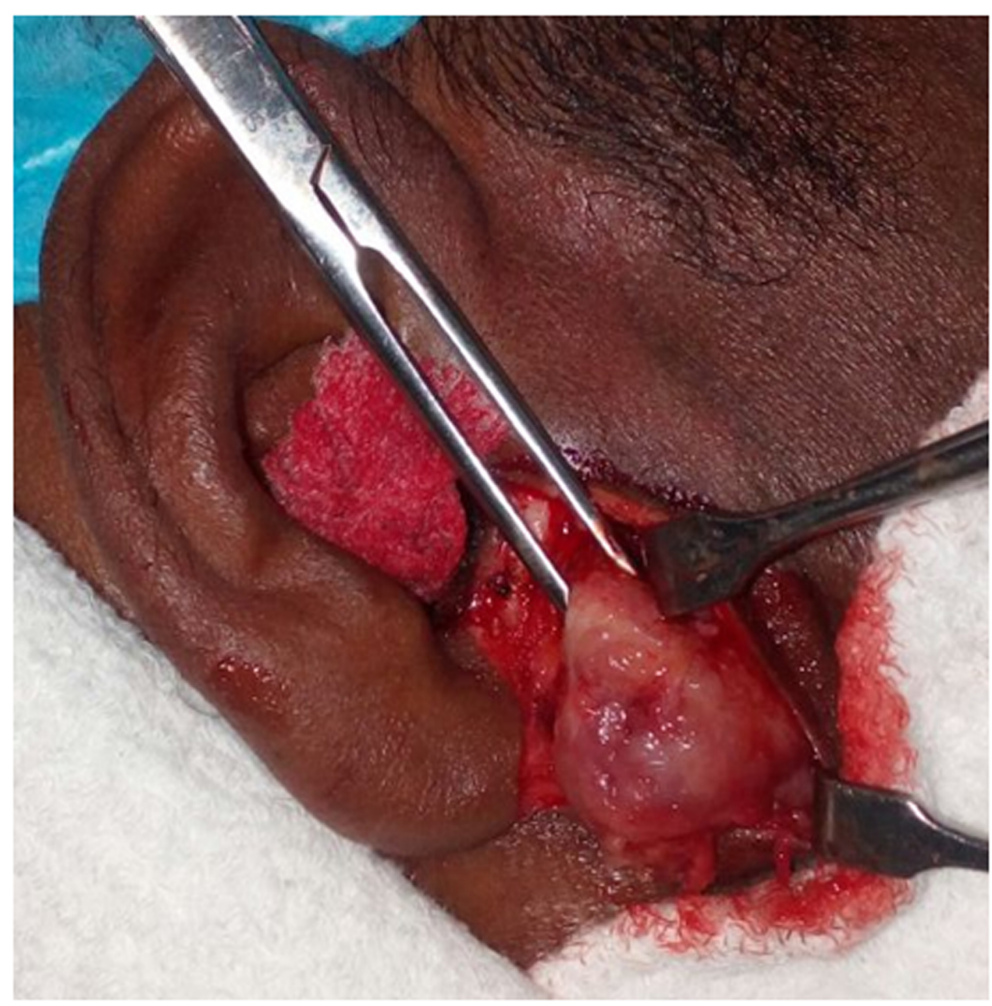

Figura 2. Abordaje endaural en el que se evidencia tumoración parotídea, que se observa unilobulada, encapsulada y vascularizada, se procede a realizar parotidectomía parcial.

Fuente: elaboración propia 


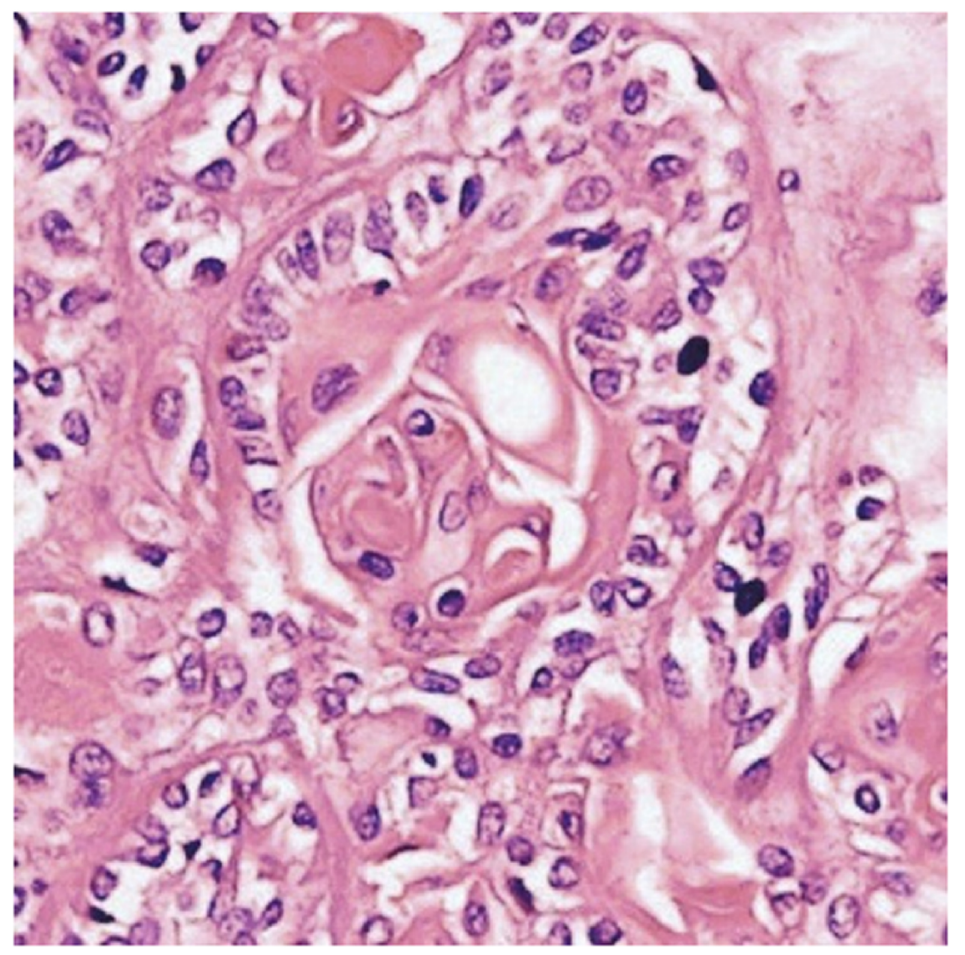

Figura 3. Corte histológico, en el que se observa neoplasia compuesta por células epiteliales que forman conductos entremezclados con células mioepiteliales, en el que se evidencia un estroma con áreas tanto mixoides como hialinizadas.

Fuente: elaboración propia

A los 3 días de procedimiento, se lleva a cabo retiro de dren tipo Penrose. A los 4 días siguientes se realiza control en el que se observa buen proceso de cicatrización, sin signos de infección o inflamación, los puntos de sutura se encuentran en posición, los cuales se decide retirar 3 días después. Se le indica rehabilitación y terapia física. Se cita 1 mes después para control, en éste se le indica una nueva cita a los 6 meses, cita en la que se observa adecuada evolución clínica y funcional, se le proporcionan citas de control cada 6 meses.

\section{Discusión}

El adenoma pleomorfo es una neoplasia de gran incidencia en cabeza y cuello, en comparación a la lesión linfoepitelial benigna, con una menor incidencia. Suelen aparecer entre la tercera y quinta década de la vida, teniendo predilección por el sexo femenino, en el caso del adenoma pleomorfo, al igual que la lesión linfoepitelial benigna asociada, además, de pacientes con VIH positivo, y es signo patognomónico 
Samuel E. Urbano Del Valle, Eilien G. Tovío Martínez, Shanny L. Miranda Duncan, 9 Jonathan Harris Ricardo

(15). Representan alrededor del 3-10\% de los tumores de la región de cabeza y cuello, además el 50 \% de las neoplasias de glándulas salivales se caracterizan por ser tumores de crecimiento lento, asintomático, de consistencia firme y cubierta por piel o mucosa sana (16).

El adenoma pleomorfo es un tumor mixto de las glándulas salivales, el cual tiene predominio por la glándula parótida, su aparición puede ser en cualquier etapa de la vida, pero es más común que se desarrolle entre la $4^{\text {ta }}$ y $6^{\text {ta }}$ década, tiene una mayor prevalencia en el sexo femenino que masculino (17). Autores como Mukai y col describieron en su estudio de 179 pacientes. De los cuales 51 fueron hombres y 128 fueron mujeres con un rango de edad entre los 16 y 84 años, al adenoma pleomorfo como la neoplasia más común de las glándulas salivales incluso en niños y adolescentes, siendo las mujeres más propensas. Los tumores de $1 \mathrm{~cm}$ de diámetro suelen tener numerosas protuberancias, lo que les confiere un aspecto lobulado, se encapsulan comúnmente en las glándulas parótidas. Añadieron que todos los tumores comparten las características diagnósticas esenciales de estar compuestos de tejidos epiteliales y mixoides, componentes que varían ampliamente, aunque uno es a menudo más predominante, la clave diagnóstica del adenoma es encontrar el componente mixoide (18). Comparándolo con el presente caso se llega a la conclusión de que lo dicho por la literatura anteriormente coincide en la edad, sitio de lesión, características clínicas e histológicas y en menor medida el género de los pacientes que, aunque su predilección es el sexo femenino, en el caso de estudio del presente artículo tuvo lugar en un paciente masculino.

Gallana y col, describen el caso de una paciente de sexo femenino de 39 años de edad con presencia de un adenoma pleomorfo localizado en hemicara izquierda en relación con pared anterior del seno maxilar izquierdo, con un crecimiento de 1 mes de evolución. Estas neoplasias representan solo el 22 \% de todas las asociadas a las glándulas, tanto mayores como menores, cuyo tratamiento fue la remoción quirúrgica (19). Tal y como se presenta en el caso actual, a diferencia de que la lesión se encontraba en lado contralateral y se trataba de género femenino.

Para poder alcanzar el diagnóstico certero de este tipo de tumores es importante evaluar las características clínicas, acompañadas siempre de exámenes complementarios como biopsia, resonancia magnética o tomografía, que confirmen que es un adenoma pleomorfo (8). En el presente caso, además del examen clínico, se utilizaron otras ayudas diagnósticas, como la tomografía computarizada, la PAAF y el estudio histopatológico.

Histopatológicamente, el adenoma pleomorfo representa una lesión pleomórfica aumentada, este pleomorfismo se debe al patrón de crecimiento del tumor, la 
diferenciación del componente epitelial y las características del estroma. Con base en la información que reporta la literatura, los componentes básicos de las células de esta clase de tumor son células dúctiles luminales y células mioepiteliales (11). Lo que permite, en el presente caso, diferenciar la lesión linfoepitelial benigna del adenoma pleomorfo. De esta forma se logra obtener un diagnóstico certero y por tanto, un plan de tratamiento ajustado al paciente.

Jain y Col presentaron el caso de una paciente de 50 años de edad con una masa de crecimiento lento, indolora en el lado izquierdo de la cara desde hace cinco años. Se observó una hinchazón multilobular ovoide bien definida con una extensión superioinferior desde el arco cigomático izquierdo hasta aproximadamente $1 \mathrm{~cm}$ por debajo del borde inferior de la mandíbula y una extensión anteroposterior desde la región media del cuerpo hasta el borde posterior de la mandíbula (18). A diferencia del presente caso cuya evolución fue de cuatro meses, el tamaño de la lesión es relativamente más pequeño ya que su tiempo de evolución fue considerablemente menor, en el examen clínico ambos mostraron asimetría facial del lado de la lesión.

\section{Conclusión}

Conocer las características clínicas y radiográficas de las lesiones que se desarrollan comúnmente en las glándulas salivales permite llevar los casos rápidamente a un tratamiento correcto y oportuno. El adenoma pleomorfo es uno de los tumores más comunes que podemos encontrar en pacientes con lesiones en glándulas salivales. Sin embargo, está la lesión linfoepitelial benigna, y aunque su aparición es común tanto en pacientes de sexo femenino, como en pacientes portadores de $\mathrm{VIH}$, se debe tener en cuenta que también se han reportado casos en pacientes $\mathrm{VIH}$ negativos, y deben considerarse como un diagnóstico diferencial en las neoplasias benignas de las glándulas salivales.

Aunque la intervención quirúrgica es el tratamiento de elección en ambas patologías, es imperativo realizar un exhaustivo estudio clínico, imagenológico e histopatológico de la posible lesión, y así descartar otras entidades que requieran un plan de tratamiento totalmente distinto, evitando cometer errores.

\section{Revelación ética}

Protección de sujetos humanos y animales. 
Samuel E. Urbano Del Valle, Eilien G. Tovío Martínez, Shanny L. Miranda Duncan, 11 Jonathan Harris Ricardo

Los autores declaran que no se realizaron experimentos en humanos o animales para este estudio.

\section{Confidencialidad de los datos}

Los autores declaran que han seguido los protocolos de su centro de trabajo sobre la publicación de datos de pacientes.

\section{Derecho a la privacidad y consentimiento informado}

Los autores han obtenido el consentimiento informado por escrito de los pacientes o sujetos mencionados en el artículo. El autor correspondiente está en posesión de este documento.

\section{Referencias}

1. Alves V, Pérez M, de Castro J, Vieira C, Leão J, Perez D. Intraoral Pleomorphic Adenoma in Young Patients. Journal of Craniofacial Surgery. 2018; 29(2): e209-e211.

2. Pillai S, Agarwal AC, Mangalore AB, Ramaswamy B, Shetty S. Benign Lymphoepithelial Cyst of the Parotid in HIV Negative Patient. J Clin Diagn Res. 2016;10(4): MD05-MD06.

3. Álvarez E, Botero X, Ardila M. Adenoma pleomórfico benigno del paladar: presentación de un caso. AMC. 2013; 17(4): 497-505.

4. Ahamed A, Kannan V, Velaven K, Sathyanarayanan G, Roshni J, Elavarasi E. Lymphoepithelial cyst of the submandibular gland. J Pharm Bioallied Sci. 2014; 6(Suppl 1): S185-S187.

5. Singh A, Kumar N, Sharma P, Singh S. Pleomorphic adenoma involving minor salivary glands of upper lip: A rare phenomenon. Journal of cancer research and therapeutics. 2015; 11(4): 1025.

6. Sánchez J, Franco L, Ochoa J. Adenoma pleomorfo de la glándula Parótida tratados quirúrgicamente, experiencia de 7 años. Archivos del Hospital Universitario" General Calixto García”. 2019; 7(1): 28-32. 
7. Castañeda A, Hernández M. Lesión linfoepitelial benigna relacionada con el VIH en un paciente pediátrico: informe de un caso. Anales de Radiología, México. 2019; 17(4): 293-301.

8. Tarsitano A, Pizzigallo A, Giorgini F, Marchetti C. Giant pleomorphic adenoma of the parotid gland: an unusual case presentation and literature review. Acta Otorrinolaringol Ital. 2015; 35(4): 293-296.

9. Valstar M, De Ridder M, Van Den Broek EC, Stuiver MM, Van Dijk BAC, Van Velthuysen LF, et al. Salivary gland pleomorphic adenoma in the Netherlands: A nationwide obserational study of primary tumor incidence, malignant transformation, recurrence, and risk factors for recurrence. Oral Oncol. 2017; 66: 93-99.

10. Joshi J, Shah S, Agarwal D, Khasgiwal A. Benign lymphoepithelial cyst of parotid gland: Review and case report. J Oral Maxillofac Pathol. 2018; 22(Suppl 1): S91-S97.

11. Enescu A, Enescu A, Bălăşoiu, M, Ciolofan M, Cápitanescu A. Histopathological study of pleomorphic adenoma of salivary glands. Rom J Morphol Embryol. 2014; 55(3): 1149-1153.

12. Khan M, Raza SS, Zaidi SAH, Hussain AK, Nadeem MD, Farid K. Pleomorphic adenoma of minor salivary glands. J Ayub Med Coll Abbottabad. 2016; 28(3): 620-622.

13. Basu S. y Patra T. Pleomorphic Adenoma of Submandibular gland. Journal of Case Reports. 2018;8(2):98-101.

14. Yáñez R, Loyola F, Maíz C, Mariangel P, Cornejo J, Martínez R, et al. tratamiento quirúrgico de los tumores de parótida: experiencia de 10 años. Revista Chilena de Cirugía. 2014; 66(3): 245-250

15. Uz U, Celik O. Pleomorphic Adenoma of the Posterior Surface of the Soft Palate Causing Sleep Disturbance: A Case Report. Am J Case Rep. 2017; 18: 1266-1270.

16. Koyama M, Terauchi T, Koizumu M, Tanaka H, Sato Y. Metastasizing pleomorphic adenoma in the multiple organs: A case report on FDG-PET/CT imaging. Medicine (Baltimore). 2018; 97(23): e11077.

17. Velpula N, Annam SR, Pallepati SR, Kumar R, Kumar A. Pleomorphic Adenoma of Cheek Masquerading as Fibrolipoma - Case Report with Review. J Clin Diagn Res. 2015; 9(11): ZD13- ZD15. 
Samuel E. Urbano Del Valle, Eilien G. Tovío Martínez, Shanny L. Miranda Duncan, 13 Jonathan Harris Ricardo

18. Mukai H, Motoori K, Horikoshi T, Takshima H, Nagai Y, Okamoto Y, et al. Adenoma de células basales de la glándula parótida; Características de la RM y diferenciación del adenoma pleomórfico. Dentomaxillofac Radiol. 2016; 45(4): 20150322.

19. Gallana S, Mayorga F, Herce J, Díaz M. Adenoma pleomorfo intraoral. Revista Española de Cirugía Oral y Maxilofacial. 2006; 28(1): 63-66.

20. Jain S, Hasan S, Vyas N, Shah N, Dalal S. Pleomorphic Adenoma of the Parotid Gland: Report of a Case With Review of Literature. Ethiop J Health Sci. 2015; 25(2): 189-194. 